\title{
An improved translational switch for long term maintenance of synaptic plasticity
}

\author{
Animesh Agarwal ${ }^{1,2}$, Naveed Aslam ${ }^{1}$, Harel Z Shouval ${ }^{1,2^{*}}$ \\ From Nineteenth Annual Computational Neuroscience Meeting: CNS*2010 \\ San Antonio, TX, USA. 24-30 July 2010
}

Memory lasts a lifetime, yet the physiological substrate of memory, synaptic contacts, are composed of proteins that have much shorter lifetimes. A physiological analog of memory formation, long-term potentiation (LTP), has a late protein synthesis dependent phase (LLTP) that can last for many hours in slices, or even days in vivo $[1,2]$. Our previous studies show that maintenance of L-LTP and memory can be accounted by persistent regulation of on-site synthesis of plasticity-related proteins by a self-sustaining regulation of translation. It has been shown that a $\alpha$ CaMKII-CPEB1 molecular pair can act as a bistable switch with different total amounts of $\alpha$ CaMKII in potentiated and nonpotentiated synapses [3].

The molecular interaction model in our previous study comprised $\alpha$ CaMKII which could be in an inactive, active and active and phosphorylated forms together with a translation regulating molecule CPEB1, which can be in an active or inactive form. The model included both degradation and new protein synthesis of $\alpha$ CaMKII. We have showen that this model is bistable [3]. The bistability was caused by interaction of $\mathrm{Ca}^{2}$ ${ }^{+}$-Calmodulin dependent and auto-phosphorylation activation, spontaneous degradation and synthesis loops of $\alpha$ CaMKII. This model could successfully account for maintenance of L-LTP over a long period of time and also proposes an explanation for why application of protein synthesis and $\alpha$ CaMKII inhibitors at induction and maintenance phases of L-LTP result in very different outcomes [3-5]

However, the protein synthesis loop in our previous model was very simplistic. Here, we suggest a more detailed model of translation with explicit implementation of mRNA and poly-ribosome concentration in the pre-synaptic spine. We assume that activated CBEB1activates mRNA which then binds preferentially to polyribosome, as compared to a non-active mRNA, for $\alpha$ CaMKII synthesis. We show that this system can act as a bistable switch. We also look at the behavior of this system at low poly-ribosome and mRNA concentration levels using stochastic simulations with Gillespie algorithm.

\section{Author details}

'Department of Neurobiology \& Anatomy, University of Texas Medical School, Houston, TX 77030, USA . ²Department of Biomedical Engineering the University of Texas, Austin, TX 78712, USA.

\section{Published: 20 July 2010}

\section{References}

1. Bliss TV, Collingridge GL: A synaptic model of memory: longterm potentiation in the hippocampus. Nature 1993, 361:31-39.

2. Feng TP: The involvement of PKC and multifunctional CaM kinase II of the postsynaptic neuron in induction and maintenance of long-term potentiation. Prog Brain Res 1995, 105:55-63.

3. Naveed Aslam, Yoshi Kubota, David Wells, Shouval ZHarel: Translational switch for long-term maintenance of synaptic plasticity. Molecular Systems Biology 2009, 5:284.

4. Otmakhov N, Griffith LC, Lisman JE: Postsynaptic inhibitors of calcium/ calmodulin-dependent protein kinase type II block induction but not maintenance of pairing-induced long-term potentiation. J Neurosci 1997, 17:5357-5365.

5. Sanhueza M, Mclntyre CC, Lisman JE: Reversal of synaptic memory by Ca2 +/calmodulin-dependent protein kinase II inhibitor. J Neurosci 2007, 27:5190-5199.

doi:10.1186/1471-2202-11-S1-P186

Cite this article as: Agarwal et al:: An improved translational switch for long term maintenance of synaptic plasticity. BMC Neuroscience 2010 11(Suppl 1):P186.

* Correspondence: harel.shouval@uth.tmc.edu

'Department of Neurobiology \& Anatomy, University of Texas Medical School, Houston, TX 77030, USA 